\title{
The Effects of Oxidative Stress in Urinary Tract Infection During Pregnancy
}

\author{
Pinar Ciragill, ${ }^{1}$ Ergul Belge Kurutas, ${ }^{2}$ Mustafa Gul, ${ }^{1}$ Metin Kilinc, ${ }^{2}$ Murat Aral, ${ }^{1}$ and Alanur Guven ${ }^{3}$ \\ ${ }^{1}$ Department of Clinical Microbiology, Faculty of Medicine, Kahramanmaras Sutcu Imam University, 46050 Kahramanmaras, Turkey \\ ${ }^{2}$ Department of Biochemistry, Faculty of Medicine, Kahramanmaras Sutcu Imam University, 46050 Kahramanmaras, Turkey \\ ${ }^{3}$ Department of Gynecology and Obstetrics, Faculty of Medicine, \\ Kahramanmaras Sutcu Imam University, 46050 Kahramanmaras, Turkey
}

Received 10 May 2005; accepted 30 June 2005

\begin{abstract}
The purpose of this study was to determine the effect of urinary tract infection (UTI) on antioxidant systems and lipid peroxidation (LPO) levels during pregnancy. We also investigated if these antioxidant systems and LPO levels differed in each trimester. One hundred forty-three nonpregnant women, as a control group, and 77 pregnant women were included in the study. Urine cultures were performed according to standard techniques. Catalase (CAT), superoxide dismutase (SOD), and LPO levels were measured using a spectrophotometer. UTI was observed in 14 of 77 pregnant women and the isolated microorganisms were Escherichia coli, Klebsiella pneumoniae, and Staphylococcus saprophyticus. CAT, SOD, and LPO levels were increased in pregnant women compared with nonpregnant women $(P<.01)$. CAT, SOD activities, and LPO levels were increased from the first trimester to the third trimester in pregnancy without UTI. However, CAT and SOD activities were decreased, LPO levels were increased from the first trimester to the third trimester in pregnancy with UTI $(P<.01)$. Pregnancy causes oxidative stress and also UTI during pregnancy may aggravate oxidative stress.
\end{abstract}

\section{INTRODUCTION}

Free radical production (superoxide, hydroxyl and nitric oxide, and other reactive oxygen species (ROS)) occurs as a consequence of the endogenous reactions and plays an important role in the cell. Increased formation of ROS and/or decreased antioxidant defense can be defined as oxidative stress, which is widely recognized as an important feature of many diseases such as diabetes mellitus, cancer, and renal failure. Superoxide dismutase (SOD) and catalase (CAT) are some important endogenous antioxidants, which protect cells from oxidative stress [1].

Lipid peroxidation (LPO) is one of the most important expressions of oxidative stress induced by ROS. Malondialdehyde (MDA) is an indicator of LPO which increases in various diseases. This increase is reflected in enhanced extraction of several MDA derivatives in the urine. It was suggested that urinary MDA levels were increased such as thallassemia, renal failure, and pancreatic disease $[2,3]$.

Pregnancy, mostly because of the mitochondria-rich placenta, is a condition that favors oxidative stress $[4,5]$.

Correspondence and reprint requests to Pinar Ciragil Department of Clinical Microbiology, Faculty of Medicine, Kahramanmaras Sutcu Imam University, 46050 Kahramanmaras, Turkey; pinarciragil@yahoo.com
In generally, immune functions reduce during pregnancy, and clinical as well as silent infections are more common than in the nonpregnant state. Urinary tract infection (UTI) is the second most common medical complication of pregnancy [6]. To our knowledge, none of the previous researches investigated MDA levels and the activities of antioxidant enzymes (CAT, SOD) in UTI during pregnancy as a response to oxidative stress. Therefore, the aim of this study was to investigate the effect of UTI on CAT and SOD activities and MDA levels in urine during pregnancy. And also it was investigated if these antioxidant enzymes and MDA differed in each trimester.

\section{METHODS}

\section{Patients and urine samples}

One hundred forty-three nonpregnant women as a control group, and 77 pregnant women (without chronic illness such as hypertension and diabetes mellitus) with symptoms suggesting acute UTI, who had been admitted to the Gynecology and Obstetric Clinic of Sutcu Imam University, were included in the study. UTI inclusion criteria were dysuria, frequency, urgency, and abdominal/flank pain with or without fever. Patients receiving antibiotic therapy were excluded from the study. Of the pregnant women $21(27.3 \%)$ were in the first trimester, $26(33.8 \%)$ in the second trimester, and $30(38.9 \%)$ in the 
third trimester. Midstream urine samples were obtained by using clean-catch technique and transmitted to Microbiology Laboratory in about half an hour. All samples were inoculated into the MacConkey and 5\% sheep blood agar and incubated at $37^{\circ} \mathrm{C}$ for 18 hours. Urinary isolates from positive cultures were identified by using API ID $32 \mathrm{E}$ (bioMérieux, France).

\section{Biochemical analysis}

Spot and 24 hour urine samples were used for biochemical analyses. To control the urine concentration, data were normalized to urine creatinine concentration. Urinary creatinine was measured in 24 hour urine samples by DADE Behring-Dimension RXL autoanalyzer (Germany).

The urine samples were diluted with 1:50 physiologic serum $(0.9 \% \mathrm{NaCl})$ for biochemical analysis. SOD activity was measured according to the method described by Fridovich [7]. CAT activity was determined by measuring the decrease in hydrogen peroxide concentration at $230 \mathrm{~nm}$ by the method of Beutler [8]. LPO level in the urine samples was expressed by MDA. It was measured according to procedure of Ohkawa et al [9].

\section{Statistical analysis}

Statistical analyses were carried out with the SPSS$\mathrm{X}$ (release 4.1) program. The $t$ test was used to analyze the difference between nonpregnant and pregnant women, for the activities of CAT, SOD and MDA levels. Also, ANOVA test was used to analyze the difference between each parameter according to trimesters in urine samples, while the differences between pregnant women with and without UTI for each parameter according to each trimester were analyzed by Mann-Whitney-U tests.

\section{RESULTS}

Pregnant women and the control group (non pregnant women) were of similar ages. It was observed that, of the pregnant women, $14(18.2 \%)$ had UTI but others had not UTI. Of the urine samples in which bacterial growth was observed, five were in the first trimester, four in the second trimester, and five in the third trimester. Bacterial distribution according to trimesters was as follows: three $E$ coli and two K pneumonaie in the first trimester; three $E$ coli and one $S$ saprophyticus in the second trimester; and three E coli and two $S$ saprophyticus in the third trimester.

Antioxidant enzymes (CAT, SOD) and MDA levels in pregnant women were increased compared to the nonpregnant women (Figure 1). In view of the trimesters, CAT, SOD, and MDA levels were increased from the first trimester to the third trimester in pregnancy without UTI. However, it was observed that CAT and SOD activities were decreased and MDA level was increased from the first trimester to the third trimester in pregnant women with UTI, compared to pregnant women without UTI $(P<.01)$ (Figure 1).

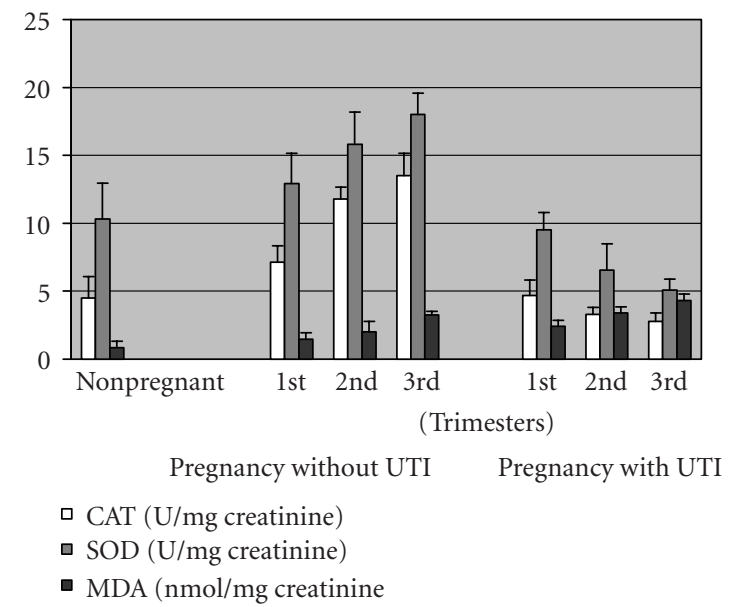

Figure 1. CAT: catalase, SOD: superoxide dismutase, MDA: malondialdehyde significant differences between nonpregnant women and pregnant women $(P<.01)$ were found. CAT, SOD and MDA levels; significant differences between pregnancy with and without UTI in each trimester were found $(P<.01)$. CAT, SOD and MDA levels; significant differences among trimesters of pregnancy with and without UTI were found $(P<.01)$.

\section{DISCUSSION}

Free radical production occurs continuously in the cell during metabolism. These radicals (hydroxyl, superoxide anion, nitric oxide, etc) are in part toxic to cell and cell membranes; however, they are normally controlled by countervailing biologic mechanisms. Severe oxidative stress produces ROS and induces uncontrolled LPO [13].

Pregnancy is a condition exhibiting increased susceptibility to oxidative stress and oxidative disorders occur, when the balance of the system of oxidants and antioxidants changes for the good of oxidant systems [4]. So, pregnancy is a physiological occasion primarily causing oxidative stress. Our results also demonstrated that MDA levels in urine were significantly elevated in pregnant women compared to nonpregnant women, with increased antioxidant enzyme activities. This may be due to increased reproductive problems in pregnancy.

LPO is also induced in placenta during pregnancy. Lipid peroxides originating from both the trophoblast and the villous core compartments are secreted into the maternal effluent, possibly adding to levels in the maternal blood as additional peroxidation cascades are initiated $[10,11]$. Nakai et al [12] reported that lipid peroxide levels in the first trimester of pregnancy were sometimes higher and sometimes lower than those in the nonpregnant control group. In the same study it was showed that, in the second trimester, MDA levels were 10 to $50 \%$ higher than the first trimester MDA levels, as usually seen; the third trimester levels sometimes, but not always, declined. In our study, MDA levels in the third trimester were significantly higher than those in the first and second trimesters of pregnancy without UTI. This result was the same in pregnancy with UTI (Figure 1). We found that 
urinary antioxidant enzymes (CAT, SOD) were gradually increased in pregnant women without UTI from the first trimester to the third trimester, as a response to oxidative stress. To the contrary, CAT and SOD activities were decreased and MDA levels were increased from the first trimester to the third trimester of pregnancy with UTI indicating that, UTI with pregnancy may aggravate oxidative stress.

The protective antioxidant mechanisms are complex and multifactorial. The susceptibility of cells to oxidative stress is a function of the overall balance between the degree of oxidative stress and the antioxidant defense capability. It is possible that, during gestation, the increase in antioxidant enzyme activities occurs in response to the normal oxidative stress due to pregnancy. At present, the nature of this mechanism is not known.

ROS are produced by activated phagocytes, which are microbicidal and can inadvertently cause tissue damage [13]. The phagocytes use ROS as a defense against foreign microorganisms. A defense mechanism that is a vital part of the immune system can also be destructive. Many inflammatory diseases are characterized by tissue damage produced by ROS which are controlled by a defense system that depends on the activity of enzymes and other nonenzyme substances. The imbalance between ROS and the body's defense system is called oxidative stress. Scavengers of ROS are important in treating and preventing the damage caused by such oxidative stress [13]. Increased lipid peroxidation and decreased CAT and SOD activities were found in pregnant women with UTI compared with those without UTI. The ratio lipid peroxidation/total antioxidant activity (MDA/CAT+SOD) was increased in pregnancy with UTI and without UTI, which indicates that oxidative stress occurs. This may be due to increased cell turnover or decreased antioxidant free radical scavenging mechanisms.

In conclusion, pregnancy causes oxidative stress from the first trimester to the third trimester; also UTI may aggravate oxidative stress during pregnancy.

\section{REFERENCES}

[1] Koracevic D, Koracevic G, Djordjevic V, Andrejevic $S$, Cosic V. Method for the measurement of antioxidant activity in human fluids. J Clin Pathol. 2001;54(5):356-361.

[2] Kang HK, Kim DK, Lee BH, et al. Urinary N-acetylbeta-D-glucosaminidase and malondialdehyde as a markers of renal damage in burned patients. J Korean Med Sci. 2001;16(5):598-602.

[3] Okur H, Kose O, Kula M, Ozturk F, Muhtaroglu S, Sumerkan B. The role of infection and free oxygen radical damage in reflux nephropathy: an experimental study. J Urol. 2003;169(5):1874-1877.

[4] Casanueva E, Viteri FE. Iron and oxidative stress in pregnancy. J Nutr. 2003;133(5 suppl 2):1700-1708.
[5] Page KR. The Physiology of Human Placenta. London, UK: UCL Press Limited; 1993:164-169.

[6] Gravett MG, Sampson JE. Other infectious conditions in pregnancy. In: James DK, Ster PJ, Weiner CP, Gonik B, eds. High Risk Pregnancy Management Options. London UK: WB Saunders; 1999:559-563.

[7] Fridovich I. Superoxide radical: an endogenous toxicant. Annu Rev Pharmacol Toxicol. 1983;23:239-257.

[8] Beutler E. Red Cell Metabolism. New York, NY: Grune and Stratton Company; 1975:261-265.

[9] Ohkawa H, Ohishi N, Yagi K. Assay for lipid peroxides in animal tissues by thiobarbituric acid reaction. Anal Biochem. 1979;95(2):351-358.

[10] Walsh SW, Wang Y. Secretion of lipid peroxides by the human placenta. Am J Obstet Gynecol. 1993;169(6):1462-1466.

[11] Poranen AK, Ekblad U, Uotila P, Ahotupa M. The effect of vitamin $\mathrm{C}$ and $\mathrm{E}$ on placental lipid peroxidation and antioxidative enzymes in perfused placenta. Acta Obstet Gynecol Scand. 1998;77(4):372-376.

[12] Nakai A, Oya A, Kobe H, et al. Changes in maternal lipid peroxidation levels and antioxidant enzymatic activities before and after delivery. J Nippon Med Sch. 2000;67(6):434-439.

[13] Freeman BA, Crapo JD. Biology of disease: free radicals and tissue injury. Lab Invest. 1982;47(5):412426. 


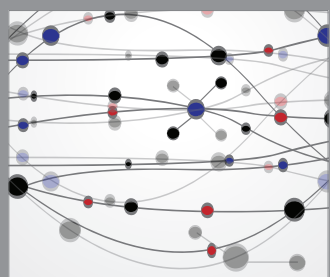

The Scientific World Journal
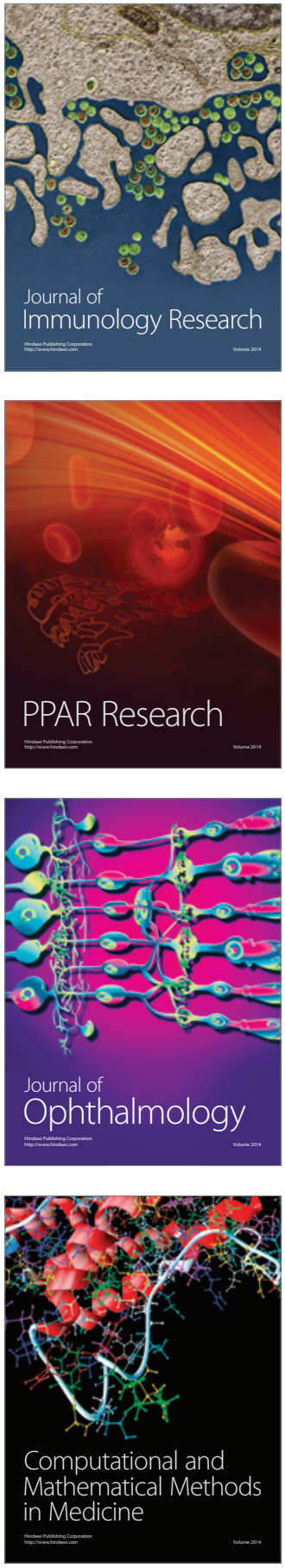

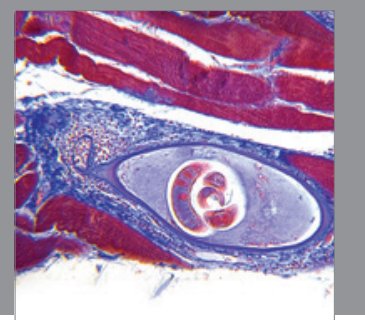

Gastroenterology

Research and Practice
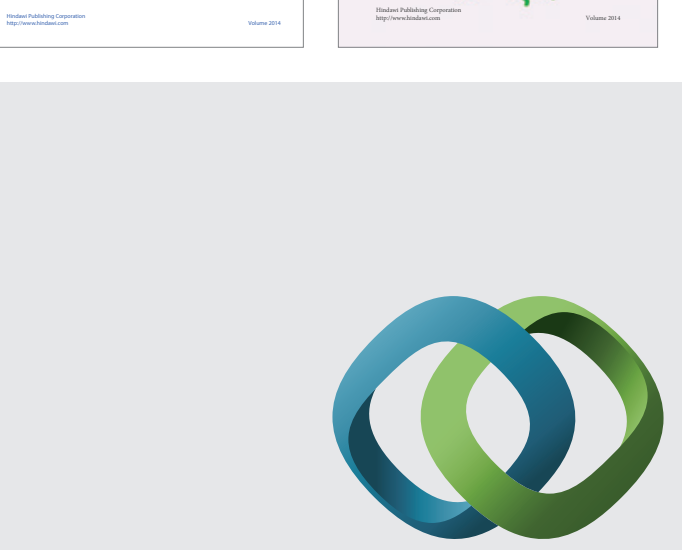

\section{Hindawi}

Submit your manuscripts at

http://www.hindawi.com
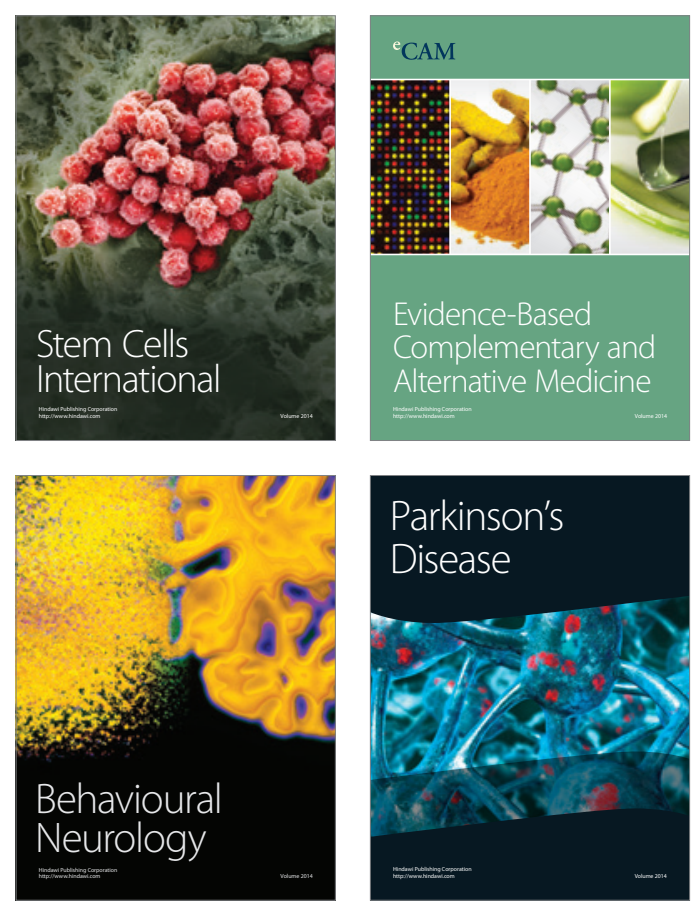

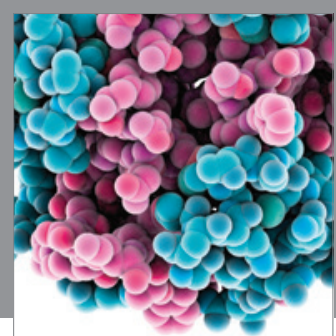

Journal of
Diabetes Research

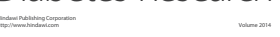

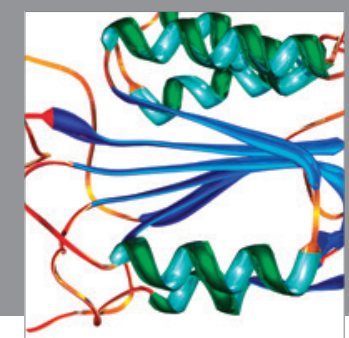

Disease Markers
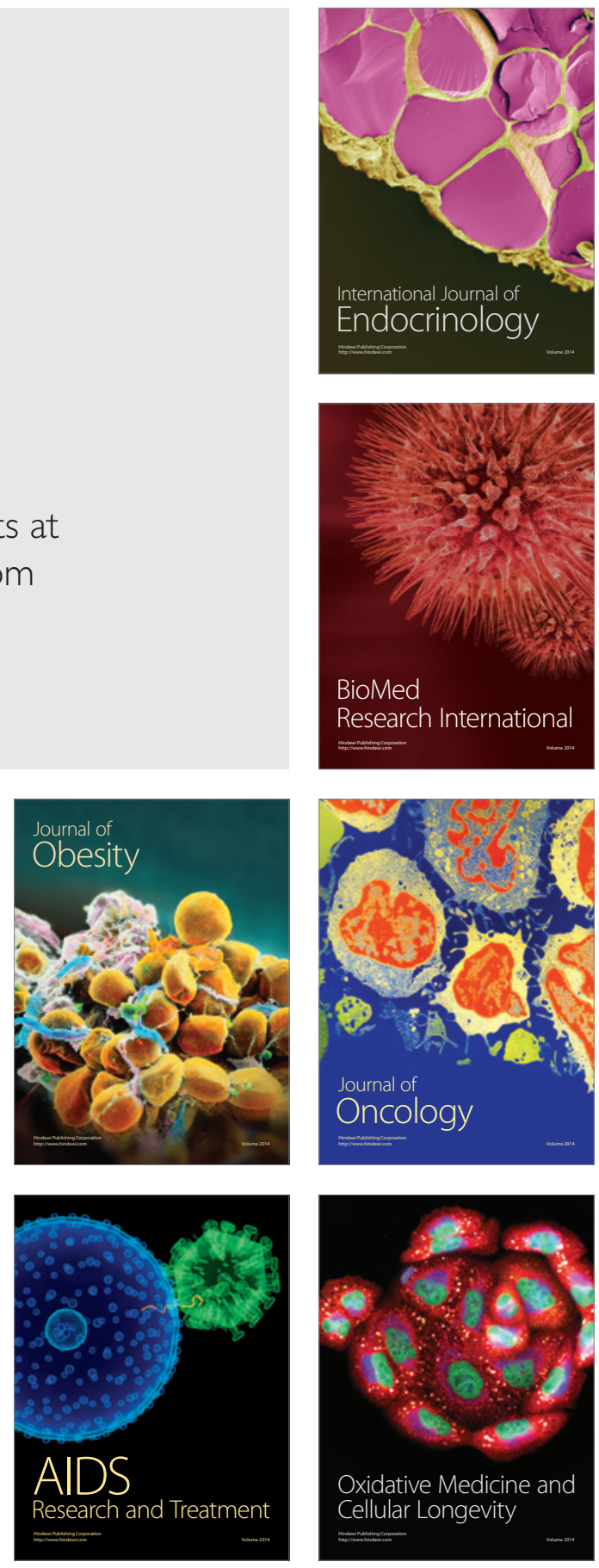